\title{
A CAMERON-MARTIN TRANSLATION THEOREM FOR A GAUSSIAN MEASURE ON $C(Y)$
}

\section{J. D. KUELBS ${ }^{1}$}

1. Introduction. Let $Y$ be the product space $\prod_{k=1}^{\infty}\left[a_{k}, b_{k}\right]$ where $-\infty<a_{k}<b_{k}<\infty$ for $k=1,2, \cdots$ and $b_{k}-a_{k}=O\left(2^{-k}\right)$. The topology on $Y$ is the product topology and $C(Y)$ denotes the space of real valued continuous functions on $Y$ with the uniform topology. Then a Gaussian measure $m$ can be defined on $C(Y)$ which is a generalization of Wiener measure and an analogue and generalization of the Cameron-Martin translation theorem [1], [2], [3] for Wiener integrals is obtainable in this setting. In another paper this measure and translation theorem will be used to obtain a representation for additive functionals on $C(X)$ when $X$ is a compact metric space.

2. The definition of the measure $m$ on $C(Y)$ and other preliminaries. If $p_{1}, \cdots, p_{k}$ are points in $Y$ such that $p_{i}=\left(c_{i 1}, c_{i 2}, \cdots\right)$ for $i=1, \cdots, k$ then $F^{p(1, \cdots, k)}$ will denote the $k$-variate Gaussian distribution with mean zero and covariance matrix $V=\left(v_{i j}\right)$ where $2 v_{i j}=\prod_{n=1}^{\infty}\left[1+\left(c_{i n}-a_{n}\right) \wedge\left(c_{j n}-a_{n}\right)\right]$ and by $a \wedge b$ we mean $\min (a, b)$. That $V$ is actually a covariance matrix follows immediately from the fact that the matrix $D_{m}=\left(d_{i j m}\right)$ with

$$
d_{i j m}=1+\left(c_{i m}-a_{m}\right) \wedge\left(c_{j m}-a_{m}\right)
$$

is nonnegative definite for $m=1,2, \cdots$ and that $V$ is the Hadamard product of the $D_{m}[4]$.

If $p_{1}=\left(c_{1}, c_{2}, \cdots\right)$ and $p_{2}=\left(d_{1}, d_{2}, \cdots\right)$ are in $Y$ and the Gaussian random variable with distribution $F^{p(i)}$ is denoted by $Z_{p(i)}$ for $i=1,2$ then $E\left[Z_{p(1)}-Z_{p(2)}\right]^{2} \leqq C \sum_{k=1}^{\infty}\left|d_{k}-c_{k}\right|$ if $\sum_{k=1}^{\infty}\left(b_{k}-a_{k}\right) C$ $\geqq \exp \left\{2 \sum_{k=1}^{\infty}\left(b_{k}-a_{k}\right)\right\}$. Therefore, using the final remark and Theorem 1 of [5], we see that these conditions on the family $F^{p(1, \cdots, k)}$, $p_{i} \in Y$, are sufficient to assure the existence of a measure $\mu$ on the Borel sets $\mathrm{e}$ of $C(Y)$ whose finite-dimensional distributions consist of the family postulated. The triple $(C(Y), a, m)$ will denote the measure space obtained from the standard completion of $(C(Y), \mathcal{C}, \mu)$.

By an $n$-dimensional rectangular subset $\rho$ of $Y$ we mean a finite set of points of $Y$ which is formed by taking the product of finite subsets of the first $n$ coordinates of $Y$ with the remaining coordinates all being $\alpha_{n}=\left(a_{n+1}, a_{n+2}, \cdots\right)$. That is, if $a_{k} \leqq x_{1}<\cdots<x_{M_{k}} \leqq b_{k}$

Received by the editors October 15, 1966.

1 Supported by NSF Grant GP-3483. 
for $k=1, \cdots, n$ then the rectangular subset formed is $\rho=\{p \in Y$ : $\left.p=\left(x_{i_{1}}, \cdots, x_{i_{n}}, \alpha_{n}\right), i_{k}=1, \cdots, M_{k}\right\}$.

If $\beta$ is an $n$-dimensional rectangular subset of $Y$ as given above then we define

$$
Q(\odot, u)=\sum_{i_{1}=1}^{M_{1}} \cdots \sum_{i_{n}=1}^{M_{n}} \frac{\left[\Delta_{1} \cdots \Delta_{n} u\left(i_{1}, \cdots, i_{n}\right)\right]^{2}}{\Delta_{i_{1}} x^{1} \cdots \Delta_{i_{n}} x^{n}}
$$

where

$$
\begin{aligned}
& \text { (a) } \Delta_{j} x^{k}=x_{j}^{k}-x_{j-1}^{k} \text { with } x_{0}^{k}=a_{k}-1 \\
& \text { for } k=1, \cdots, n \text { and } j=1, \cdots, M_{k}, \\
& \text { (b) } \Delta_{1} \cdots \Delta_{k} u\left(i_{1}, \cdots, i_{n}\right)=\Delta_{1} \cdots \Delta_{k-1} u\left(i_{1}, \cdots, i_{k}, \cdots, i_{n}\right) \\
& -\Delta_{1} \cdots \Delta_{k-1} u\left(i_{1}, \cdots, i_{k}-1, \cdots, i_{n}\right) \text { for } k=2, \cdots, n, \\
& \\
& \Delta_{1} u\left(i_{1}, \cdots, i_{n}\right)=u\left(i_{1}, \cdots, i_{n}\right)-u\left(i_{1}-1, \cdots, i_{n}\right), \\
& \text { and we assume } u\left(i_{1}, \cdots, i_{n}\right)=0 \text { if some } i_{j}=0 .
\end{aligned}
$$

Let $W(\odot, u)=C(\odot)^{-1 / 2} \exp \{-Q(\odot, u)\}$ where

$C(\odot)=\left[\Delta_{1} x^{1} \cdots \Delta_{M_{1}} x^{1}\right]^{L_{1}} \cdots\left[\Delta_{1} x^{n} \cdots \Delta_{M_{n}} x^{n}\right]^{L_{n}} \pi^{L}, \quad L=\prod_{j=1}^{n} M_{j}$,

and $L_{k}=L / M_{k}$.

From these definitions several things follow. First we notice that the variable $u\left(i_{1}, \cdots, i_{n}\right)$ is associated with the point $\left(x_{i_{1}}, \cdots, x_{i_{n}}, \alpha_{n}\right)$ of $\beta$ and that for any $\rho$ the function $W(\rho, u)$ is a Gaussian density of $L$ variables. Secondly, it can be shown that if $F^{P}$ denotes the Gaussian distribution function corresponding to the density $W(\odot, u)$ then $F^{\mathcal{P}}$ is identical to $F^{p(1, \cdots, L)}$ where $p_{1}, \cdots, p_{L}$ are the points in $\mathcal{P}$. Furthermore, if $Z\left(i_{1}, \cdots, i_{n}\right)$ denotes the Gaussian random variable associated with the point $\left(x_{i_{1}}, \ldots, x_{i_{n}}, \alpha_{n}\right)$ and $\Delta_{1} \ldots$ $\Delta_{n} Z\left(i_{1}, \cdots, i_{n}\right)$ is defined as was $\Delta_{1} \cdots \Delta_{n} u\left(i_{1}, \cdots, i_{n}\right)$ in $(2.2)$ then the $\Delta_{1} \cdots \Delta_{n} Z\left(i_{1}, \cdots, i_{n}\right)$ are independent Gaussian random variables with expectation zero and variance $\frac{1}{2} \Delta_{i_{1}} x^{1} \cdots \Delta_{i_{n}} x^{n}$ for $i_{k}=1, \cdots, M_{k} ; k=1, \cdots, n$.

3. A statement of the main result. Let $Y_{n}=\left\{\prod_{k=1}^{n}\left[a_{k}, b_{k}\right]\right\} \times \alpha_{n}$ for $n=1,2, \cdots$ and by $S_{n}$ denote the $2^{n}$ subsets of $Y_{n}$ formed by selecting $n-k$ of the first $n$ coordinates and setting each such $x_{j}$ equal to $a_{j}$ while the remaining $k$ coordinates among the first $n$ are allowed to vary as they do in $Y_{n}$. The symbol $S$ denotes $\bigcup_{n=1}^{\infty} S_{n}$. If $I \in S$ and $I$ has $k>0$ coordinates which vary then $\mu_{I}$ denotes Lebesgue measure 
on $I$ when $I$ is considered as $k$ dimensional. If $I$ is the single point $\alpha_{0}=\left(a_{1}, a_{2}, \cdots\right)$ then $\mu_{I}$ is the measure obtained by placing mass one at this point.

The Borel sets of $Y$ are denoted by $B$ and if $B \in B$ we define $\nu(B)$ $=\sum_{I \in S} \mu_{I}(B \cap I)$. Then $\nu$ is sigma-additive on $B, \nu(Y)=\sum_{I \in S} \mu_{I}(I)$ $=\prod_{k=1}^{\infty}\left(1+b_{k}-a_{k}\right)$, and $C(Y)$ is dense in $\mathfrak{L}_{2}(Y)$ with respect to mean square convergence. In fact, polynomials in finitely many coordinates of $Y$ with rational coefficients are dense in $\mathfrak{L}_{2}(Y)$ and, as a result, a countable orthonormal basis of polynomials exists for $\mathfrak{L}_{2}(Y)$.

If $g$ is in $\mathscr{L}_{2}(Y)$ and $f_{0}(p)=\int_{Y(p)} g d \nu$ where $Y(p)=\prod_{k=1}^{\infty}\left[a_{k}, x_{k}\right]$ for $p=\left(x_{1}, x_{2}, \cdots\right)$ in $Y$ then $f_{0} \in C(Y)$. By $\int_{Y} g d \tilde{f}$, when $g$ is $\mathfrak{L}_{2}(Y)$, we will mean a generalization of the Paley, Wiener, and Zygmund integral as given in [6]. The precise definition of $\int_{Y} g d \tilde{f}$ occurs later. We now state our main result. It is easy to see how it generalizes the results of [1] and [3].

Theorem. Let $F$ be a measurable functional. If $g$ is in $\mathfrak{L}_{2}(Y)$ and $f_{0}(p)=\int_{Y(p)} g d \nu$ for all $p$ in $Y$ then

$$
E(F(f))=\exp \left\{-\int_{Y} g^{2} d \nu\right\} E\left\{F\left(f+f_{0}\right) \exp \left\{-2 \int_{Y} g d \bar{f}\right\}\right\}
$$

in the sense that if either integral exists both exist and they are equal. The symbol $E(\cdot)$ denotes integration with respect to the measure $m$.

4. The following lemmas are needed in the proof of our theorem. The definitions and results involving Riemann-Stieltjes integration of functions of $n$-variables can be found in [3, pp. 412-415].

If $I \in S$ and $I$ has $n$ coordinates which vary then $\tau(I)=\bigcup_{k=0}^{n} \tau_{k}(I)$ where $\tau_{k}(I)$ is the collection of $k$-dimensional subsets of $I$ formed by choosing $n-k$ of the $n$ coordinates of $I$ which vary and setting each $x_{j}$ of this collection equal to $a_{j}$ or $b_{j}$ while each $x_{j}$ of the remaining $k$ coordinates which can vary satisfies $a_{j} \leqq x_{j} \leqq b_{j}$.

If $f$ is defined on $Y$ and $J \in \tau(I)$ for some $I \in S$ then $f$ is of bounded variation on $J$ if $f^{J}$ is of bounded variation there when $J$ is considered as being finite dimensional. Here by $f^{J}$ we mean $f$ restricted to $J$. The Riemann-Stieltjes integral of $g$ with respect to $f$ over $J, \int_{J} g d f$, when $f$ and $g$ have domain $Y$ is simply the Riemann-Stieltjes integral of $g^{J}$ with respect to $f^{J}$ over $J$. When $J$ is a single point $\alpha$ it is understood that $\int_{J} g d f=g(\alpha) f(\alpha)$.

By $\boldsymbol{P}_{N}$ we will denote the $n$-dimensional rectangular subset formed by the sets $L_{k}=\left\{x_{j}=a_{k}+\left(b_{k}-a_{k}\right)(j-1) / N\right\}$ where $j=1, \cdots, N+1$ and $k=1, \cdots, n$. The point $\left(x_{i_{1}}, \cdots, x_{i_{n}}, \alpha_{n}\right) \in \mathcal{P}_{N}$ will be denoted 
by $\left(i_{1}, \cdots, i_{n}\right)$ and if $f \in C(Y)$ we define $\Delta_{1} \cdots \Delta_{n} f\left(i_{1}, \cdots, i_{n}\right)$ as in (2.2) with $f\left(i_{1}, \cdots, i_{n}\right)$ replacing $u\left(i_{1}, \cdots, i_{n}\right)$. Finally, if $f_{0}, f \in C(Y)$ we define

$$
\begin{aligned}
& H\left(\odot_{N}, f_{0}, f\right) \\
& =\sum_{\left(i_{1}, \cdots, i_{n}\right) \in \odot_{N}} \frac{\left[\Delta_{1} \cdots \Delta_{n} f_{0}\left(i_{1}, \cdots, i_{n}\right) \Delta_{1} \cdots \Delta_{n} f\left(i_{1}, \cdots, i_{n}\right)\right]}{\Delta_{i_{1}} x^{1} \cdots \Delta_{i_{n}} x^{n}}
\end{aligned}
$$

where $\Delta_{j} x^{k}$ is as in (2.2). The next lemma now follows directly using results found in [3, pp. 412-415].

Lemma 1. Let $g \in C(Y)$ be of bounded variation on all elements of $\tau(I)$ for all $I \in S$. Then if $f_{0}(p)=\int_{Y(p)} g d \nu$ for $p \in Y$ we have that

$$
\lim _{N \rightarrow \infty} H\left(\odot_{N}, f_{0}, f\right)=\sum_{I \in S_{n}} \int_{I} g d f
$$

where the convergence is bounded in $N$ for all $f$ in any uniformly bounded subset of $C(Y)$.

If $F$ is a functional on $C(Y)$ which has a Gaussian distribution with respect to the measure $m$ we will write $F=\mathfrak{l}\left(\mu, \sigma^{2}\right)$ where $\mu$ and $\sigma^{2}$ are the expectation and variance of $F$.

Lemma 2. If $g$ satisfies the conditions of Lemma 1 then the functionals $\left\{\int_{I} g d f: I \in S\right\}$ form an independent family and $\int_{I} g d f=\mathfrak{N}\left(0, \frac{1}{2} \int_{I} g^{2} d \mu_{I}\right)$. Moreover, $G(f)=\sum_{I \in S} \int_{I} g d f$ exists for almost all $f$ and $G(f)$ $=\Re\left(0, \frac{1}{2} \int_{Y} g^{2} d \nu\right)$.

Proof. If $I \in S$ and $Z_{N}(f, I)$ is a Riemann-Stieltjes sum for $\int_{I} g d f$ then using the final remark of $\$ 2$ we see that $Z_{N}(f, I)$ is a linear combination of independent Gaussian functionals each with zero expectation. Moreover, the variance of $Z_{N}(f, I)$ is a Riemann sum for $\frac{1}{2} \int_{I} g^{2} d \mu_{I}$. Hence $\int_{I} g d f=\Re\left(0, \frac{1}{2} \int_{I} g^{2} d \mu_{I}\right)$ for $I \in S$. The independence of the family $\left\{\int_{I} g d f: I \in S\right\}$ is also assured because of the independence mentioned at the end of $\$ 2$. Finally, the distribution of $G(f)$ follows because it is the sum of a sequence of independent Gaussian functionals each with expectation zero and such that the sum of their variances is $\frac{1}{2} \int_{Y} g^{2} d \nu$ which is finite.

We will denote $\sum_{I \in S} \int_{I} g d f$ by $\int_{Y} g d f$ provided the sum exists for almost all $f$ in $C(Y)$. Let $\left\{\phi_{k}(p)\right\}$ be a complete orthonormal set of functions for $\mathscr{L}_{2}(Y)$ each of which satisfies the conditions on $g$ in Lemma 1 for $I \in S$. Let $g$ be in $\mathfrak{L}_{2}(Y)$ and suppose $g_{n}(p)=\sum_{k=1}^{n} c_{k} \phi_{k}(p)$ where $c_{k}=\int_{Y} g \phi_{k} d \nu$. Under these conditions we have the following lemma. 
Lemma 3. The generalized Stieltjes integral $\int_{Y} g d \tilde{f}=\lim _{n \rightarrow \infty} \int_{Y} g_{n} d f$ exists for almost all $f \in C(Y)$ and $\int_{Y} g d \tilde{f}=\mathfrak{l}\left(0, \frac{1}{2} \int_{Y} g^{2} d \nu\right)$.

Proof. Now $\lim _{n \rightarrow \infty} \int_{Y} g_{n} d f=\sum_{k=1}^{\infty} c_{k} \int_{Y} \phi_{k} d f$ where $c_{k}=\int_{Y} g \phi_{k} d \nu$ and $c_{k} \int_{Y} \phi_{k} d f=\mathfrak{N}\left(0, c_{k}^{2} / 2\right)$. Furthermore, since $\left\{\phi_{k}(p)\right\}$ is an orthogonal set we see that $\left\{c_{k} \int_{Y} \phi_{k} d f\right\}$ is an independent family. Therefore, since $\sum_{k=1}^{\infty} c_{k}^{2}=\int_{Y} g^{2} d \nu$ is finite it follows that

$$
\int_{Y} g d \tilde{f}=\sum_{k=1}^{\infty} c_{k} \int_{Y} \phi_{k} d f=\mathfrak{N}\left(0, \frac{1}{2} \int_{Y} g^{2} d \nu\right) .
$$

The definition of $\int_{Y} g d \tilde{f}$ for $g$ in $\mathfrak{L}_{2}(Y)$ follows that used in [6] to define a generalized Stieltjes integral. Furthermore, if $g$ satisfies the conditions of Lemma 1 for all $I \in S$ it is easily shown that $\int_{Y} g d \tilde{f}$ $=\int_{Y} g d f$ for almost all $f \in C(Y)$. It is also true that $\int_{Y} g d \tilde{f}$ is essentially independent of the orthonormal basis of $\mathfrak{L}_{2}(Y)$ used in its definition as long as each element of the basis satisfies the conditions of Lemma 1 for all $I \in S$.

5. Proof of theorem. Our proof will first be for the case that $g$ satisfies Lemma 1 and $F$ is a bounded continuous functional which vanishes if $\|f\|=\max _{y \in Y}|f(y)|>M$. Let $J_{n}$ be the continuous map of $C(Y)$ into $C(Y)$ such that $J_{n}(f)\left(x_{1}, \cdots, x_{n}, x_{n+1}, \cdots\right)$ $=f\left(x_{1}, \cdots, x_{n}, \alpha_{n}\right)$ for all $\left(x_{1}, x_{2}, \ldots\right)$ in $Y$ and define $F_{n}(f)$ $=F\left(J_{n}(f)\right)$. Let $\left\{\rho_{N}\right\}$ be the sequence of $n$-dimensional rectangular subsets used in Lemma 1 and by $\left\{G_{N}\right\}$ we will mean a corresponding sequence of linear continuous mappings of $C(Y)$ into $C(Y)$ such that $G_{N}(f)$ depends only on the values of $f$ at points in $\mathcal{P}_{N}$. We also assume $\lim _{N \rightarrow \infty} G_{N}(f)=f$ on $Y_{n}$. The construction of a sequence $\left\{G_{N}\right\}$ is obtained through use of the techniques found in [5]. Now using the translation theorem for Lebesgue integrals on $R_{(N+1)^{n}}$

$$
\begin{aligned}
E\left\{F_{n}\left(G_{N}(f)\right)\right\}= & \exp \left\{-Q\left(\odot_{N}, f_{0}\right)\right\} \\
& \cdot E\left\{F_{n}\left(G_{N}\left(f+f_{0}\right)\right) \exp \left\{-2 H\left(\odot_{N}, f_{0}, f\right)\right\}\right\}
\end{aligned}
$$

where $H\left(\mathcal{P}_{N}, f_{0}, f\right)$ is defined as in (4.1). Letting $N$ approach infinity and using Lemma 1 along with the bounded convergence theorem we have

$$
\begin{aligned}
E\left\{F_{n}(f)\right\}= & \exp \left\{-\sum_{I \in S_{n}} \int_{I} g^{2} d \mu_{I}\right\} \\
& \cdot E\left\{F_{n}\left(f+f_{0}\right) \exp \left\{-2 \sum_{I \in S_{n}} \int_{I} g d f\right\}\right\}
\end{aligned}
$$


Furthermore, using Lemma 2 it follows that

$$
\left\{F_{n}\left(f+f_{0}\right) \exp \left\{-2 \sum_{I \in S_{n}} \int_{I} g d f\right\}\right\}
$$

is a $\mathscr{L}_{\mathbf{1}}$ fundamental sequence which converges almost everywhere to $F\left(f+f_{0}\right) \exp \left\{-2 \int_{Y} g d f\right\}$ and, letting $n$ approach infinity in (5.1), we have that (3.1) holds for $F$ and $g$ as given.

Now let $g$ be in $\mathscr{L}_{2}(Y)$ and $\left\{\phi_{k}(p)\right\}$ be an orthonormal basis for $\mathscr{L}_{2}(Y)$ such that each $\phi_{k}$ satisfies Lemma 1 for all $I \in S$. Let $f_{n}(p)$ $=\int_{Y(p)} \sum_{k=1}^{n} c_{k} \phi_{k} d \nu$ where $c_{k}=\int_{Y} \phi_{k} g d \nu$. Then $\left\{f_{n}\right\}$ converges to $f_{0}$ in the uniform topology and by the previous case we have for all $n$ that

$$
\begin{aligned}
E(F(f))= & \exp \left\{-\int_{Y}\left(\sum_{k=1}^{n} c_{k} \phi_{k}\right)^{2} d \nu\right\} \\
& \cdot E\left\{F\left(f+f_{n}\right) \exp \left\{-2 \sum_{k=1}^{n} \int_{Y} c_{k} \phi_{k} d f\right\}\right\} .
\end{aligned}
$$

Since the sequence of functionals integrated above converges almost surely to $F\left(f+f_{0}\right) \exp \left\{-2 \int_{Y} g d \tilde{f}\right\}$ and it is also $\mathfrak{L}_{1}$ fundamental we have, by letting $n$ approach infinity, that (3.1) holds for $F$ a bounded continuous functional which vanishes if $\|f\|>M$. The extension to arbitrary measurable $F$ follows as usual so the proof is complete.

\section{BIBLIOGRAPHY}

1. R. H. Cameron and W. T. Martin, Transformations of Wiener integrals under translations, Ann. of Math. 45 (1944), 386-396.

2. R. H. Cameron and R. E. Graves, Additive functionals on a space of continuous functions. I, Trans. Amer. Math. Soc. 70 (1951), 160-176.

3. J. Yeh, Cameron-Martin translation theorems in the Wiener space of functions of two variables, Trans. Amer. Math. Soc. 107 (1963), 409-420.

4. M. Marcus and N. A. Khan, A note on the Hadamard product, Canad. Math. Bull. 2 (1959), 81-83.

5. J. D. Kuelbs, Measures on $C(Y)$ when $Y$ is a compact metric space, Proc. Amer. Math. Soc. 18 (1967), 248-254.

6. R. E. A. C. Paley, N. Wiener and A. Zygmund, Notes on random functions, Math. Z. 37 (1933), 647-668.

UNIVERSITY OF Wisconsin 